Outcomes and prognosticators in T4 RCC patients who underwent RN or CN

\title{
Outcomes and prognosticators of stage 4 renal cell carcinoma with pathological T4 primary lesion using a large Canadian multi-institutional database
}

Justin D. Oake ${ }^{1}$; Premal Patel ${ }^{2}$; Luke T. Lavallée ${ }^{3}$; Jean-Baptiste Lattouf ${ }^{4}$; Olli Saarela ${ }^{5}$; Laurence Klotz ${ }^{6}$; Ronald B. Moore ${ }^{7}$; Anil Kapoor ${ }^{8}$; Antonio Finelli ${ }^{6}$; Ricardo A. Rendon ${ }^{9}$; Jun Kawakami $^{10}$; Alan So ${ }^{11}$; Darrel E. Drachenberg ${ }^{1}$

${ }^{1}$ Section of Urology, University of Manitoba, Winnipeg, MB, Canada; ${ }^{2}$ Department of Urology, University of Miami, Miami, FL, United States; ${ }^{3}$ Division of Urology, University of Ottawa, ON, Canada; ${ }^{4}$ Section of Urology, University of Montreal, Montreal, QC, Canada; ${ }^{5}$ Dalla Lana School of Public Health, University of Toronto, Toronto, ON, Canada; ${ }^{6}$ Division of Urology, University of Toronto, Toronto, ON, Canada; ${ }^{7}$ Division of Urology, University of Alberta, Edmonton, AB, Canada; ${ }^{8}$ Division of Urology, McMaster University, Hamilton, ON, Canada; ${ }^{9}$ Department of Urology, Dalhousie University, Halifax, NS, Canada; ${ }^{10}$ Division of Urology, University of Calgary, Calgary, AB, Canada; ${ }^{11}$ Department of Urologic Sciences, University of British Columbia, Vancouver, BC, Canada

Cite as: Can Urol Assoc J 2019 July 23; Epub ahead of print. http://dx.doi.org/10.5489/cuaj.5941

Published online July 23, 2019

$* * *$

\section{Abstract}

Introduction: The primary objective of this study was to evaluate outcomes and prognosticators in patients who underwent radical nephrectomy (RN) or cytoreductive nephrectomy (CN), depending on the clinical stage of disease preoperatively, with a pathological T4 (pT4) renal cell carcinoma (RCC) outcome. There is little data on the outcome of this specific subset of patients. Methods: From 2009-2016, we identified patients in the Canadian Kidney Cancer information system (CKCis) who underwent RN or CN and were found to have pT4 RCC. Clinical, operative, and pathological variables were analyzed with univariable and multivariable Cox proportional hazard models to identify factors associated with overall survival (OS). Survival curves were created using Kaplan-Meier methods and compared using the log-rank test.

Results: A total of 82 patients were included in the study cohort. Median patient age was 62 years (interquartile range [IQR] 55, 70). The majority of patients had clear-cell histology, 50 (61\%), and 14 (17\%) had sarcomatoid characteristics. Median followup was 12 months (IQR 3, 24). At last followup, eight (10\%) patients are alive with no evidence of disease, 27 (33\%) are alive with disease, four (5\%) were lost to followup, 36 (44\%) died of disease, and seven (8\%) died of other causes. Tumor histologic subtype (clear-cell vs. non-clear-cell) $(\mathrm{p}=0.0032)$, larger 
tumor size $(\mathrm{cm})(\mathrm{p}=0.012)$, and Fuhrman grade (G4 vs. G2-G3) ( $\mathrm{p}=0.045)$ were significantly associated with mortality in a multivariable Cox regression model.

Conclusions: For patients with pT4 RCC after RN or CN, survival is poor. Sarcomatoid features, non-clear-cell histology, and presence of systemic symptoms were associated with worse OS.

\section{Introduction}

In 2017, there were an estimated 63,990 new cases of kidney cancer diagnosed in the United

States, with 14,400 estimated deaths. ${ }^{1}$ While renal cell carcinoma (RCC) stage migration has resulted in more than $50 \%$ of patients being diagnosed with AJCC stage I disease, largely attributed to the increased use of cross-sectional imaging, nearly $20 \%$ of patients are still diagnosed with pathologic stage 4 disease, and pathologic stage 4 disease has remained relatively constant over time. ${ }^{2}$ Despite advances in systemic therapies, survival rates for patients with locally advanced disease are poor, with the 10-year cancer specific survival (CSS) and median CSS for pathological T4 (pT4) disease at $11.6 \%$ and 0.9 years, respectively. ${ }^{3}$

While there is a lack of evidence supporting the use of neoadjuvant therapy in locally advanced kidney cancer, results of recent clinical trials evaluating the feasibility of antiangiogenic agents (vascular endothelial growth factor receptor tyrosine kinase inhibitors [VEGFr TKI]) have demonstrated a radiological response to systemic therapy, 26-32\% of patients. ${ }^{4-7}$ Adjuvant therapy with sunitinib (Sutent ${ }^{\mathrm{TM}}$ ) following nephrectomy in non-metastatic RCC was granted FDA approval in November 2017. Approval was based on the recently updated S-TRAC trial which demonstrated improved disease-free survival (DFS) in the sunitinib group. ${ }^{8}$ An updated subgroups analysis continued to demonstrate improved DFS across all subgroups as well as those with advanced locoregional disease (T3 Nx, Fuhrman grade $\geq 2$, Eastern Cooperative Oncology Group (ECOG) performance status $\geq 1$, T4 and/or nodal involvement). Median overall survival (OS) at the time of publication was not reached for the treatment or placebo arm. ${ }^{8}$ Results from the ASSURE and PROTECT trial which looked at sunitinib, sorafenib $\left({ }^{\mathrm{TM}}\right)$ and pazopanib ${ }^{\mathrm{TM}}$ ) in the adjuvant setting failed to demonstrate a difference in DFS. ${ }^{9,10}$ The different results of these trials may be explained by differences in their patient population. In S-TRAC, only T3 and T4 RCC were included and there was also a higher proportion of T4 relative to the ASSURE and PROTECT trials. Furthermore, clear cell histology was mandatory in S-TRAC, but not in ASSURE or PROTECT. Five randomized adjuvant trials currently accruing patients, evaluating immune checkpoint inhibitors (ICPIs), the PROSPER (Nivolumab $\left({ }^{\mathrm{TM}}\right)$ ) in treating patients with localized kidney cancer undergoing nephrectomy) and IMmotion010 (Azetzolizumab $\left.{ }^{\mathrm{TM}}\right)$ ) as adjuvant therapy in patients with RCC following nephrectomy ${ }^{11}$, INmotion151 (Atezolizumab plus Bevacizumab $\left({ }^{\mathrm{TM}}\right)$ ) as first-line agents compared to Sunitinib 
(Motzer et al., 2018), ${ }^{12}$ CheckMate-9ER (Nivolumab plus Cabozantinib ( $\left.{ }^{\mathrm{TM}}\right)$ ) in patients who are treatment naïve with advanced or metastatic RCC (ClinicalTrials.gov, 2017), ${ }^{13}$ and KEYNOTE426 (Pembrolizumab $\left.{ }^{\mathrm{TM}}\right)$ ) in treating patients with treatment-naïve advanced/metastatic RCC (Atkins et al., 2016) ${ }^{14}$ will provide insight into the role of ICPIs as adjuvant therapy in patients with kidney cancer. CheckMate-214 (Nivolumab plus Ipilimumab $\left({ }^{\mathrm{TM}}\right)$ ) noted improved survival outcomes as compared to Sunitinib in treatment-naïve metastatic RCC (Motzer et al., 2018). ${ }^{15}$

In SURTIME, an EORTC randomized control trial, patients were randomized to sunitinib followed by cytoreductive nephrectomy (CN) and subsequent sunitinib versus upfront CN followed by sunitinib. On intention-to-treat analysis, deferred CN was non-inferior to up front $\mathrm{CN}$ - HR 0.57 favoring deferred nephrectomy ( $\mathrm{p}=0.032$ ). The data suggested that deferring $\mathrm{CN}$ was likely not detrimental in the targeted therapy era. ${ }^{16}$ CARMENA, a French randomized trial, compared metastatic RCC patients to either CN and sunitinib versus sunitinib alone. The results in the sunitinib-alone group were noninferior to those in the nephrectomy-sunitinib group with regard to overall survival - HR 0.89. ${ }^{17}$

Since pT4 RCC is relatively rare and difficult to study in large trials, we sought to evaluate the outcomes of patients with pT4 RCC using a large multi-institutional database. Our primary objective was to evaluate OS for our cohort of patients who underwent radical nephrectomy (RN) or CN for RCC and were found to have pT4 disease. Our secondary objective was to establish predictors of worse OS.

\section{Methods}

The Canadian Kidney Cancer information system (CKCis) is a collaborative multi-institutional database from 13 centers in 6 provinces and has been described previously. ${ }^{18-20}$ This dataset was initiated in 2011 and includes data entered retrospectively and prospectively for a sample of patients with renal tumors treated as of 1998. Demographic, clinical, pathological, and oncological variables are collected and the dataset is updated regularly. Institutional review board approval was obtained from each contributing site.

We identified patients in CKCis who underwent RN or CN, depending on the clinical stage of disease preoperatively, and were found to have pT4 RCC. Patients with node positivity or metastasis were not excluded. pT4 disease was defined according to the 2010 TNM cancer staging system as involvement beyond Gerota's fascia or tumor which extends into the ipsilateral adrenal gland. ${ }^{21}$ Patient information was prospectively collected from 2009 to 2016.

Demographic, clinical, operative, and pathological data were obtained. Demographic variables included age, sex, ECOG performance status, body mass index (BMI), and smoking history. Clinical and operative factors included time to nephrectomy, follow-up time, clinical TNM staging, recipients of first line adjuvant VEGFr TKI, preoperative laboratory results including hemoglobin, estimated glomerular filtration rate (eGFR), alkaline phosphatase (ALP), lactate dehydrogenase (LDH), corrected calcium, platelets, presence of systemic symptoms, presence of local symptoms including gross hematuria or flank pain, intraoperative blood loss, 
surgical approach, and operative duration. Pathologic characteristics including histologic subtype, Fuhrman grade, pathological TNM staging, tumor size, adrenal involvement, presence of sarcomatoid characteristics, positive margin status, and tumor necrosis were analyzed. As the number of deaths in our cohort was high, outcomes such as cancer specific survival were not assessed. The Kaplan-Meier method was used for OS and the log-rank test was used to test for differences. Univariable and multivariable Cox regression analysis identified predictors for mortality. Variables significant in the univariate analysis were included in the multivariable model, with the exception of those variables with large proportion of missing data. The only univariable significant predictor that was omitted from the multivariable model due to missing data was blood loss (32\% missing). Statistical significance was defined as $P<0.05$.

\section{Results}

\section{Clinical and pathological characteristics}

Overall, 2,442 patients within CKCis underwent RN since 2008. During the study period, 82 patients within the CKCis database were surgically treated for RCC and found to have pT4 disease. Adjacent organ extension included peritoneum tumor invasion (1), positive peritoneal nodule (1), omental tumor nodule (1), tumor extension into colon (1), and pancreatic invasion (2). Capsular tumor invasion (17), perinephric fat tumor invasion (53), renal vessel tumor invasion (26), and sinus fat tumor invasion (37) were also recorded. Median patient age was 62 years (IQR 55,70), 58 (71\%) were men, and 28 (34\%) patients presented with systemic symptoms. Twenty-three (28\%) patients had clinical stage T4 pre-operatively. Twenty (24\%) had clinical N1 disease, and 33 (40\%) had clinical M1 disease. The majority of patients had clear cell histology, 50 (61\%), and 14 (17\%) had sarcomatoid characteristics. Final pathology demonstrated pN1 in 27 (33\%) patients and pM1 in 32 (39\%). Of the 32 patients with pM1 disease, 6 underwent metastasectomy. The sites for metastatectomy included bone (1), lung (2), lymph node (1), pancreas (1), and other location (1). Furthermore, 13 of the 32 patients with pM1 disease received stereotactic body radiation therapy (SBRT). Sixty-nine (84\%) patients had Fuhrman grade 3 or 4, 44 (54\%) had direct adrenal extension, 28 (34\%) had regional lymph node involvement, and 29 (35\%) had a positive margin (Table 1).

\section{Survival}

After a median post-operative follow-up of 12 months (IQR 3, 24), 8 (10\%) patients were alive with no evidence of disease, 27 (33\%) are alive with disease, 4 (5\%) were lost to follow-up, 36 (44\%) died of disease, and 7 (8\%) died of other causes. As expected, patients with non-clear cell histology ( $p=0.03)$, presence of systemic symptoms $(\mathrm{p}=0.045)$, and presence of sarcomatoid characteristics ( $p=0.027$ ) (Fig. 1 ), had a significantly worse OS. Univariable Cox proportional hazard regression analyses revealed that year of nephrectomy, ECOG performance status (1 vs. 0 ), tumor histologic subtype (clear cell vs. non-clear cell), tumor size (cm), intraoperative blood 
loss (ml), Fuhrman grade (G4 vs. G2-G3), presence of systemic symptoms, and presence of sarcomatoid characteristics were significantly associated with mortality (Table 2).

In a multivariable Cox regression model including factors showing univariable association, we found that tumor histologic subtype (clear cell vs. non-clear cell) (HR 0.36, 95\% CI 0.18-0.71), tumor size (cm) (HR 1.12, 95\% CI 1.03-1.22), and Fuhrman grade (G4 vs. G2G3) (HR 2.33, 95\% CI 1.02-5.32) were significantly associated with OS (Fig. 2).

\section{Discussion}

To our knowledge, our findings represent the largest evaluation of patients who underwent RN and were found to have pT4 RCC. We have demonstrated that more than three quarters of patients (72\%) were clinically understaged compared to their final pathology. Our findings indicate that pathological T4 disease following RN has poor OS. Patients with larger tumor size, higher Fuhrman grade (G4 vs. G2-G3), or non-clear cell histology had worse survival on our multivariable analysis.

To date, only one study has evaluated outcomes and prognosticators of pathological T4 RCC. A single institutional study of 61 patients diagnosed with pT4 RCC undergoing RN found that preoperative LDH and ALP, M stage, $\mathrm{pN}$ stage, and sarcomatoid dedifferentiation were significantly associated with survival. ${ }^{22}$ However, the aforementioned study was limited by a small cohort and it was also a single institutional review. In contrast, our findings were collected from a national multi-institutional database, possibly better reflecting real world data.

Although targeted therapies have improved patients' outcomes among those with nonclear cell RCC, survival is significantly inferior compared with clear cell RCC patients. ${ }^{23,24}$ Similar to our findings, symptomatic characteristics of renal tumors have been demonstrated to be an independent prognostic factor affecting survival. ${ }^{25,26}$ In particular, tumors associated with anorexia, weakness, or symptoms of metastasis were associated with worse median OS compared to asymptomatic patients, as well as those with a symptom presentation including hematuria, lumbar pain, or a palpable mass. ${ }^{26}$ Our results for patients with sarcomatoid differentiation align with previous studies which have illustrated worse outcomes including more aggressive tumor biology, higher rates of tumor recurrence, and poor survival. ${ }^{27,28}$

Among patients with locoregional clear cell RCC, neoadjuvant systemic therapy has demonstrated consistent primary tumor size reduction. ${ }^{29}$ The potential advantages for neoadjuvant targeted therapy include making unresectable tumors resectable, surgical approach can be changed from radical to partial nephrectomy (PN), and renal tumors with inferior vena cava extension may be resected with lesser operations. In our study, only 8 (10\%) patients of the study cohort underwent preoperative systemic treatment. Lane et al. reported on 72 patients who received sunitinib before planned $\mathrm{PN}$ and reported a mean reduction of $32 \%$ in tumor volume, and this reduction occurred in 65 (83\%) tumors. ${ }^{6}$ Rini et al. evaluated 25 patients with localized clear cell RCC in a prospective phase II trial than received pazopanib for 8 to 16 weeks. ${ }^{7}$ Therapy resulted in reduction in tumor burden and enabled PN. Karam et al. reported on 24 
patients with locally-advanced non-metastatic clear cell RCC who received axitinib. ${ }^{4}$ Therapy was reportedly well tolerated and median reduction in primary renal tumor diameter was $28.3 \%$. Disease progression however, was observed in $4-8 \%$ of patients. These data suggest that in a high risk patient population with locoregional RCC, neoadjuvant therapy may reduce tumor burden and possibly the complexity of the surgery.

There are a number of limitations to consider when interpreting our results. The multiinstitutional design, although advantageous to reduce biases found within single-center studies, is also subject to heterogeneity in data collection and follow-up. We also acknowledge that clinical stage is related to imaging characteristics, which was not centrally reviewed and may influence our findings. Further, we suspect that under-reporting of T4 disease may be due to limitations of cross-sectional imaging. Nazim et al. (2011) noted that CT scan had a poor sensitivity (68\%) and positive predictive value (76\%) for capsular invasion in RCC when compared to final surgical pathology. Although all patients are offered CKCis, it doesn't capture all patients with the disease of interest as not all Urologists in Canada are involved with CKCis. Furthermore, both locally advanced and metastatic patients were included in the pT4 cohort, combining two different populations in the analysis.

\section{Conclusions}

For patients with pT4 RCC after RN, survival is poor. More than three quarters of patients (72\%) were initially clinically understaged compared to their final pathology. Sarcomatoid features, non-clear cell histology, and presence of systemic symptoms in particular were associated with worse overall survival. Although pre-surgical VEGFr TKI therapy in a high-risk RCC population, such as ours, appears to induce tumor shrinkage, future studies to evaluate the benefit of neo-adjuvant therapy in this population are still needed. For now, this approach should only be attempted in clinical trials, until further studies investigating oncologic and survival outcomes are conducted. 
Outcomes and prognosticators in T4 RCC patients who underwent RN or CN

\section{References}

1. Cancer stat facts: Kidney and renal pelvis cancer. http://seer.cancer.gov/statfacts/html/kidrp.html. Updated 2018. Accessed January 2, 2019.

2. Kane CJ, Mallin K, Ritchey J, et al. Renal cell cancer stage migration: Analysis of the national cancer data base. Cancer 2008;113:78-83.

3. Kim SP, Alt AL, Weight CJ, et al. Independent validation of the 2010 American joint committee on cancer TNM classification for renal cell carcinoma: Results from a large, single institution cohort. J Urol 2011;185:2035-39.

4. Karam JA, Devine CE, Urbauer DL, et al. Phase 2 trial of neoadjuvant axitinib in patients with locally advanced nonmetastatic clear cell renal cell carcinoma. Eur Urol 2014;66:87480 .

5. Hatiboglu G, Hohenfellner M, Arslan A, et al. Effective downsizing but enhanced intratumoral heterogeneity following neoadjuvant sorafenib in patients with non-metastatic renal cell carcinoma. Langenbecks Arch Surg 2017;402:637-44.

6. Lane BR, Derweesh IH, Kim HL, et al. Presurgical sunitinib reduces tumor size and may facilitate partial nephrectomy in patients with renal cell carcinoma. Urol Oncol 2015;33:112.e15-112.e21.

7. Rini BI, Plimack ER, Takagi T, et al. A phase II study of pazopanib in patients with localized renal cell carcinoma to optimize preservation of renal parenchyma. J Urol 2015;194:297303.

8. Motzer RJ, Ravaud A, Patard JJ, et al. Adjuvant sunitinib for high-risk renal cell carcinoma after nephrectomy: Subgroup analyses and updated overall survival results. Eur Urol 2018;73:62-68.

9. Haas NB, Manola J, Uzzo RG, et al. Adjuvant sunitinib or sorafenib for high-risk, nonmetastatic renal-cell carcinoma (ECOG-ACRIN E2805): A double-blind, placebo-controlled, randomised, phase 3 trial. Lancet 2016;387:2008-16.

10. Motzer RJ, Haas NB, Donskov F, et al. Randomized phase III trial of adjuvant pazopanib versus placebo after nephrectomy in patients with localized or locally advanced renal cell carcinoma. J Clin Oncol 2017;35:3916-23.

11. Lenis AT, Donin NM, Johnson DC, et al. Adjuvant therapy for high risk localized kidney cancer: Emerging evidence and future clinical trials. J Urol 2018;199:43-52.

12. Motzer RJ, Powles T, Atkins MB, et al. IMmotion151: a randomized phase III study of atezolizumab plus bevacizumab versus sunitinib in untreated metastatic renal cell carcinoma. 2018 Genitourinary Cancers Symposium. Abstract 578. Presented February 10, 2018.

13. ClinicalTrials.gov. 2017. Identifier NCT03141177, a study of nivolumab combined with cabozantinib compared to sunitinib in previously untreated advanced or metastatic renal cell carcinoma (CheckMate 9ER), May 4, 2017, [about 4 screens]. Bethesda: National Library of Medicine (US). https://clinicaltrials.gov/ ct2/show/NCT03141177?term=checkmate+9ER\&cond=Renal +Cell+Carcinoma\&rank=1. Accessed 15 December 2018. 
14. Atkins MB, Plimack ER, Puzanov I, et al. Axitinib in combination with pembrolizumab in patients (pts) with advanced renal cell carcinoma (aRCC): preliminary safety and efficacy results. Ann Oncol 2016;6:266 (Abstract 773PD).

15. Motzer RJ, Tannir NM, McDermott DF, et al. Nivolumab plus ipilimumab versus sunitinib in advanced renal-cell carcinoma. N Engl J Med 2018;378:1277-90.

16. Bex A, Mulders P, Jewett M, et al. Comparison of immediate vs deferred cytoreductive nephrectomy in patients with synchronous metastatic renal cell carcinoma receiving sunitinib: The SURTIME randomized clinical trial. JAMA Oncol 2018.

17. Mejean A, Ravaud A, Thezenas S, et al. Sunitinib alone or after nephrectomy in metastatic renal-cell carcinoma. $N$ Engl J Med 2018;379:417-27.

18. Patel P, Nayak JG, Liu Z, et al. A multicentered, propensity matched analysis comparing laparoscopic and open surgery for pT3a renal cell carcinoma. J Endourol 2017;31:645-50.

19. Nayak JG, Patel P, Bjazevic J, et al. Clinical outcomes following laparoscopic management of pT3 renal masses: A large, multi-institutional cohort. Can Urol Assoc J 2015;9:397-402.

20. Nayak JG, Patel P, Saarela O, et al. Pathological upstaging of clinical T1 to pathological T3a renal cell carcinoma: A multi-institutional analysis of short-term outcomes. Urology 2016;94:154-60.

21. Edge SB, Compton CC. The American joint committee on cancer: The 7th edition of the AJCC cancer staging manual and the future of TNM. Ann Surg Oncol 2010;17:1471-74.

22. Borregales LD, Kim DY, Staller AL, et al. Prognosticators and outcomes of patients with renal cell carcinoma and adjacent organ invasion treated with radical nephrectomy. Urol Oncol 2016;34:237.e19-237.e26.

23. Ciccarese C, Iacovelli R, Brunelli M, et al. Addressing the best treatment for non-clear cell renal cell carcinoma: A meta-analysis of randomised clinical trials comparing VEGFR-TKis versus mTORi-targeted therapies. Eur J Cancer 2017;83:237-46.

24. de Velasco G, McKay RR, Lin X, et al. Comprehensive analysis of survival outcomes in non-clear cell renal cell carcinoma patients treated in clinical trials. Clin Genitourin Cancer 2017;15:652-60.e1.

25. Lee CT, Katz J, Fearn PA, et al. Mode of presentation of renal cell carcinoma provides prognostic information. Urol Oncol 2002;7:135-40.

26. Patard JJ, Leray E, Rodriguez A, et al. Correlation between symptom graduation, tumor characteristics and survival in renal cell carcinoma. Eur Urol 2003;44:226-32.

27. Kim T, Zargar-Shoshtari K, Dhillon J, et al. Using percentage of sarcomatoid differentiation as a prognostic factor in renal cell carcinoma. Clin Genitourin Cancer 2015;13:225-30.

28. Merrill MM, Wood CG, Tannir NM, et al. Clinically nonmetastatic renal cell carcinoma with sarcomatoid dedifferentiation: Natural history and outcomes after surgical resection with curative intent. Urol Oncol 2015;33:166.e21-166.e29.

29. Bindayi A, Hamilton ZA, McDonald ML, et al. Neoadjuvant therapy for localized and locally advanced renal cell carcinoma. Urol Oncol 2018;36:31-7. 
Figures and Tables

Fig. 1. (A) Overall survival stratified by histology subtype (log rank $\mathrm{p}<0.05)$. (B) Overall survival stratified by presence of systemic symptoms at time of diagnosis (log rank $\mathrm{p}<0.05$ ). (C) Overall survival stratified by presence of sarcomatoid characteristics (log rank $\mathrm{p}<0.05$ ).
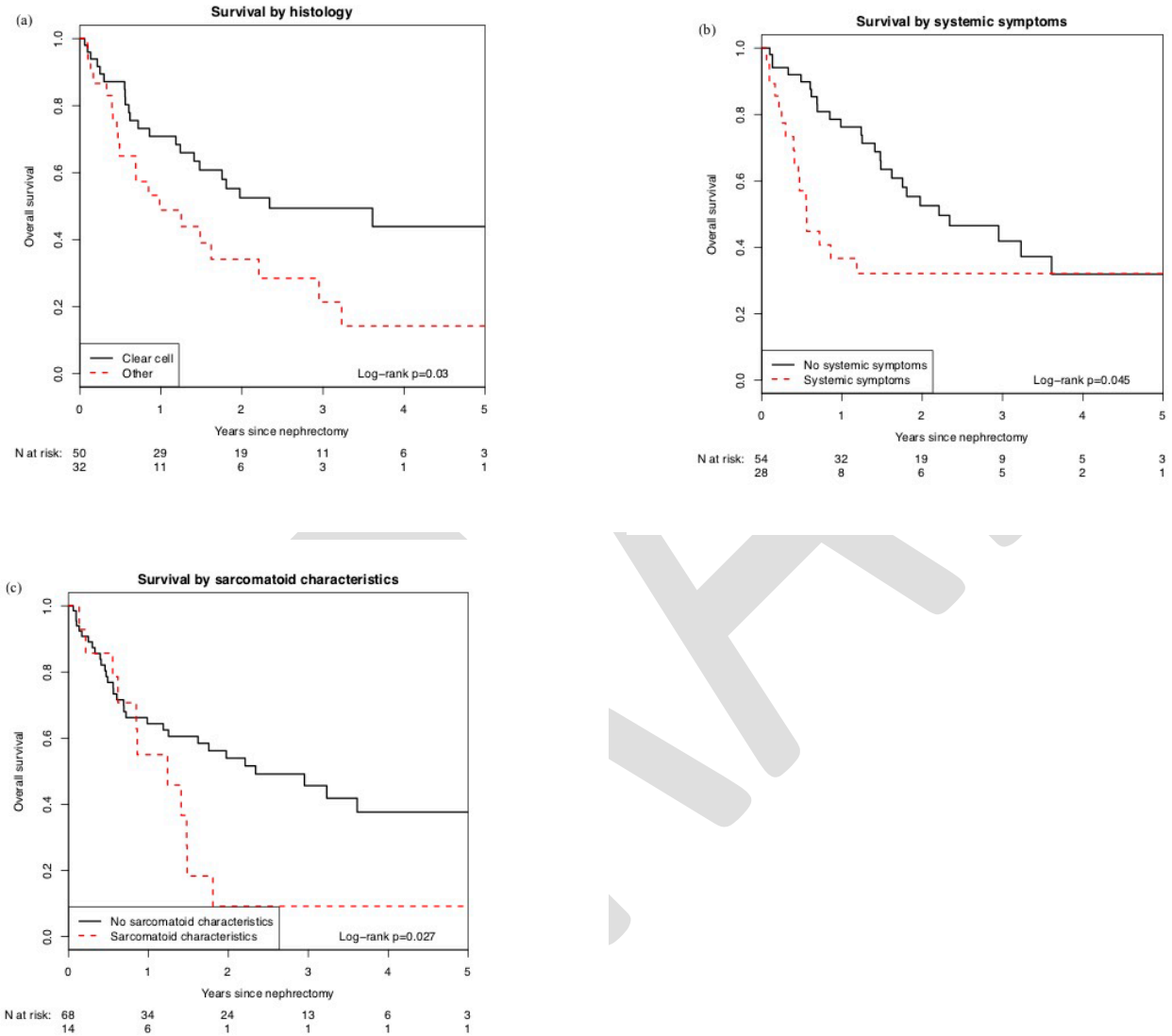
Fig. 2. Forest plot demonstrating multivariable cox regression analysis of clinical and pathological features for the postoperative prediction of overall survival in 82 patients with pT4 renal cell carcinoma treated with radical or cytoreductive nephrectomy.

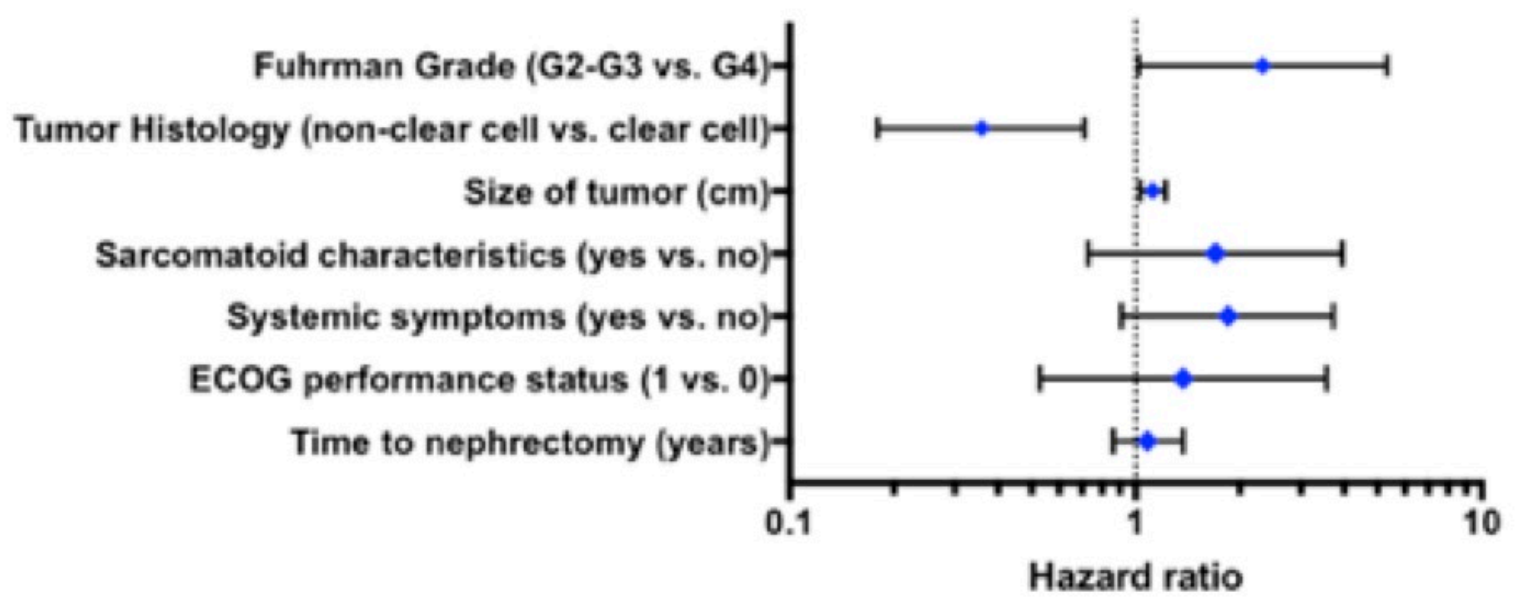


Outcomes and prognosticators in T4 RCC patients who underwent RN or CN

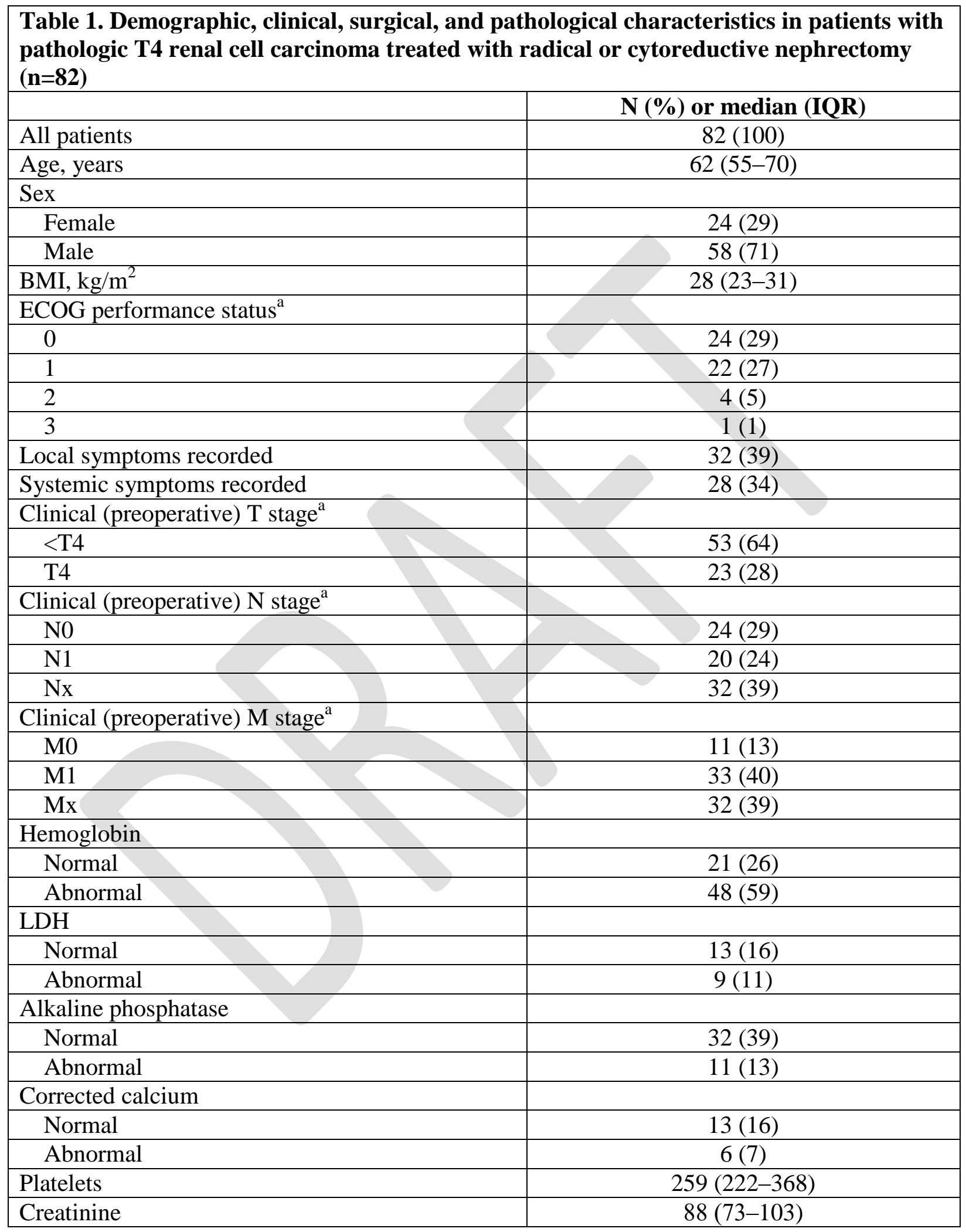


Outcomes and prognosticators in T4 RCC patients who underwent RN or CN

\begin{tabular}{|c|c|}
\hline eGFR, $\mathrm{ml} / \mathrm{min} / 1.73 \mathrm{~m}^{2}$ & $79(63-90)$ \\
\hline$>60$ & $55(79)$ \\
\hline $30-60$ & $15(21)$ \\
\hline Preoperative systemic therapy recorded & $8(10)$ \\
\hline \multicolumn{2}{|l|}{ Surgical approach } \\
\hline Lap & $13(16 \%)$ \\
\hline Open & $64(78 \%)$ \\
\hline Unknown & $5(6 \%)$ \\
\hline Tumor diameter at nephrectomy, cm & $12(9.0-13.5)$ \\
\hline \multicolumn{2}{|l|}{ Laterality } \\
\hline Bilateral & $1(1)$ \\
\hline Left & +2 \\
\hline Right & $38(46)$ \\
\hline \multicolumn{2}{|l|}{ Pathologic N stage } \\
\hline N0 & $32(39)$ \\
\hline N1 & $27(33)$ \\
\hline Nx & $23(28)$ \\
\hline Pathologic M stage & $8=$ \\
\hline M0 & +2 \\
\hline$\sqrt{1}$ & +2 \\
\hline $\mathrm{Mx}$ & +2 \\
\hline Histology & 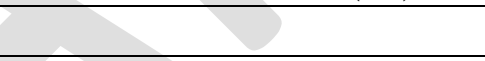 \\
\hline Clear cell & 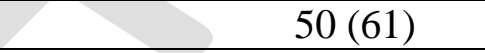 \\
\hline Nonclear cell & $32(39)$ \\
\hline \multicolumn{2}{|l|}{ Fuhrman grade $^{\mathrm{a}}$} \\
\hline 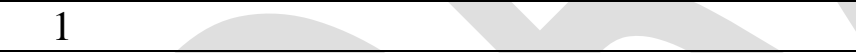 & $0(0)$ \\
\hline 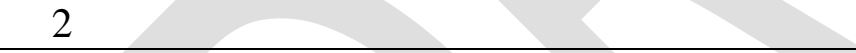 & $5(6)$ \\
\hline 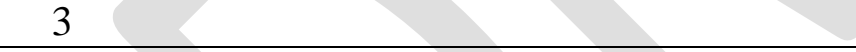 & $28(34)$ \\
\hline 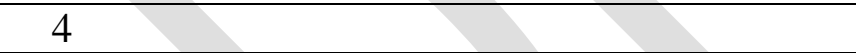 & $41(50)$ \\
\hline Sarcomatoid dedifferentiation recorded & $14(17)$ \\
\hline Necrosis recorded & $13(16)$ \\
\hline Thrombectomy recorded & $70(85)$ \\
\hline Direct adrenal extension recorded & $44(54)$ \\
\hline Positive surgical margin recorded ${ }^{\mathrm{a}}$ & $29(35)$ \\
\hline Regional lymph node involvement recorded & $28(44)$ \\
\hline
\end{tabular}

${ }^{\mathrm{a}}$ Numbers do not always add to 82 because of missing values. BMI: body mass index; ECOG:

Eastern Cooperative Oncology Group; eGFR: estimated glomerular filtration rate; IQR:

interquartile range; LDH: lactate dehydrogenase; Lap: laparoscopic. 
Outcomes and prognosticators in T4 RCC patients who underwent RN or CN

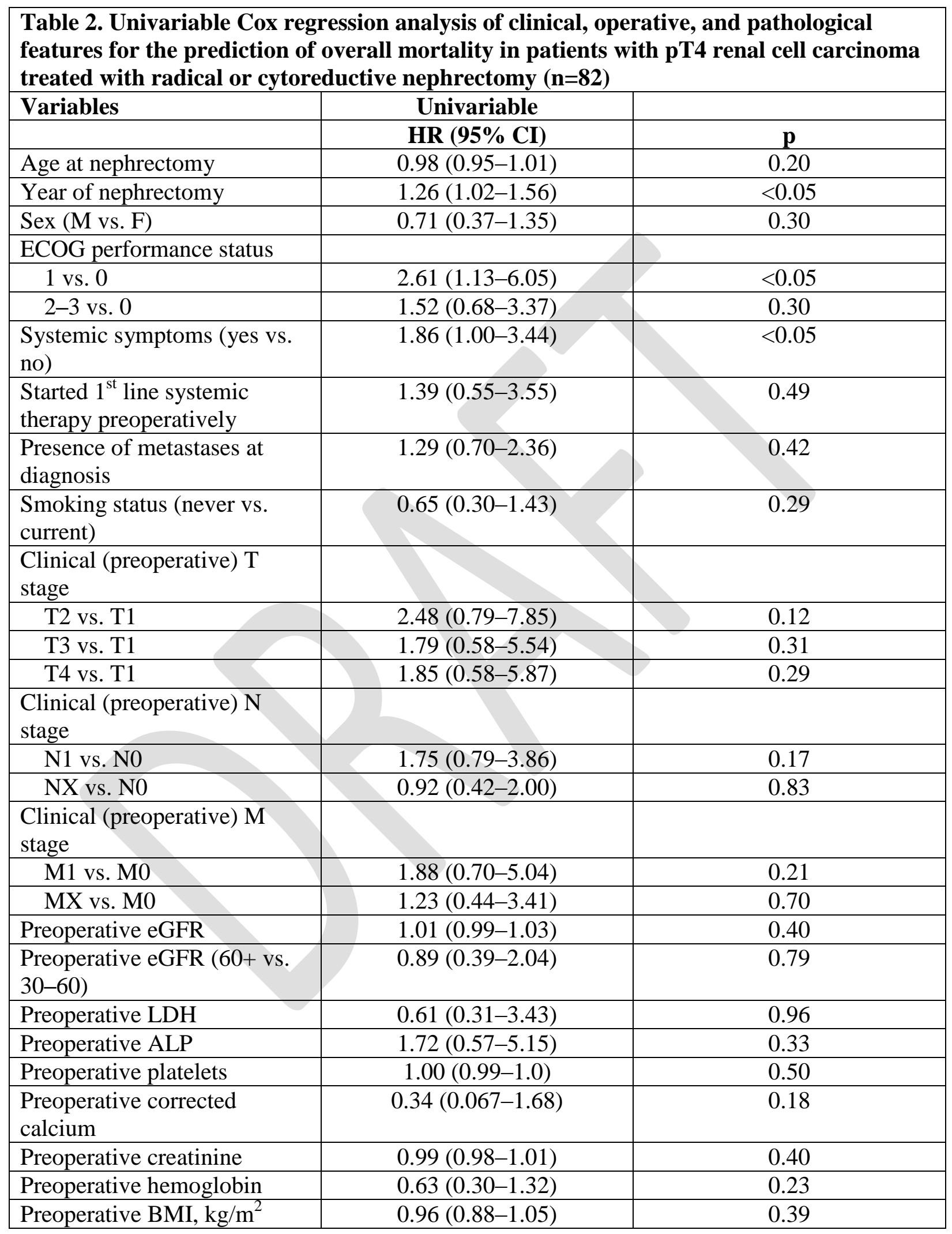


Outcomes and prognosticators in T4 RCC patients who underwent RN or CN

\begin{tabular}{|c|c|c|}
\hline \multicolumn{3}{|l|}{ ASA status } \\
\hline ASA 2 vs. 1 & $0.70(0.18-2.73)$ & 0.61 \\
\hline ASA 3 vs. 1 & $1.04(0.29-3.78)$ & 0.95 \\
\hline ASA 4 vs. 1 & $2.24(0.54-9.21)$ & 0.27 \\
\hline $\begin{array}{l}\text { Surgical approach (open vs. } \\
\text { lap) }\end{array}$ & $1.25(0.55-2.84)$ & 0.59 \\
\hline Size of tumor $(\mathrm{cm})$ & $1.12(1.04-1.21)$ & $<0.01$ \\
\hline $\begin{array}{l}\text { Histology (clear-cell vs. non- } \\
\text { clear-cell) }\end{array}$ & $1.93(1.05-3.54)$ & $<0.05$ \\
\hline \multicolumn{3}{|l|}{ Pathological nodal status } \\
\hline N1 vs. N0 & $1.36(0.66-2.80)$ & 0.40 \\
\hline Nx vs. N0 & $0.88(0.41-1.87)$ & 0.74 \\
\hline Pathological M stage & - & \\
\hline M1 vs. M0 & $1.66(0.49-5.57)$ & 0.41 \\
\hline MX vs. M0 & $0.95(0.28-3.27)$ & 0.94 \\
\hline Thrombectomy (yes vs. no) & $1.57(0.56-4.41)$ & 0.39 \\
\hline Adrenal invasion (yes vs. no) & $0.56(0.30-1.03)$ & 0.062 \\
\hline $\begin{array}{l}\text { Sarcomatoid characteristics } \\
\text { (yes vs. no) }\end{array}$ & $2.12(1.08-4.19)$ & $<0.05$ \\
\hline Tumor necrosis (yes vs. no) & $1.41(0.65-3.07)$ & 0.39 \\
\hline $\begin{array}{l}\text { Regional lymph node } \\
\text { involvement (yes vs. no) }\end{array}$ & $1.73(0.90-3.33)$ & 0.10 \\
\hline $\begin{array}{l}\text { Fuhrman grade (G4 vs. G2- } \\
\text { G3) }\end{array}$ & $3.24(1.62-6.48)$ & $<0.01$ \\
\hline $\begin{array}{l}\text { Positive margin status (yes } \\
\text { vs. no) }\end{array}$ & $1.85(0.97-3.53)$ & 0.064 \\
\hline
\end{tabular}

ALP: alkaline phosphatase; ASA: American Society of Anesthesiologists; BMI: body mass index; CI: confidence interval; ECOG: Eastern Cooperative Oncology Group; eGFR: estimated glomerular filtration rate; F: female; HR: hazard ratio; LDH: lactate dehydrogenase; Lap: laparoscopic; M: male. 